\title{
Monitorage de la qualité: l'enquête est en cours (3e partie)
}

Les médecins veulent pouvoir soigner leurs patients de la meilleure manière possible et s'engagent jour après jour en faveur de la qualité des soins, notamment par le biais de discussions de cas, de mises à jour des normes thérapeutiques et lors de formations continues. A l'aide de données chiffrées, le projet Monitorage de la qualité entend montrer l'ensemble des démarches en faveur de la qualité menées par les médecins du domaine ambulatoire. Suite au succès de la phase pilote en 2010, dix sociétés de discipline participent désormais au projet - soit deux tiers des médecins exerçant dans le domaine ambulatoire.

Varja Nikolic

Correspondance: FMH, département DDQ Elfenstrasse 18 CH-3000 Berne 15 Tél. 0313591111 Fax 0313591112 ddq[at]fmh.ch
Dans le cadre du projet Monitorage de la qualité en médecine ambulatoire dans l'ensemble de la Suisse, la FMH attache une grande importance à la collaboration avec les sociétés de discipline, ces dernières étant les mieux à même de définir leurs activités en faveur de la qualité. La direction du projet a donc tenu à donner la parole aux sociétés participant au projet. Aujourd'hui, c'est au tour des représentants des disciplines ayant récemment rejoint le projet - la gynécologie et l'obstétrique, la pneumologie, l'anesthésiologie et la réanimation, la radiologie, l'ORL et la chirurgie cervico-faciale - de nous expliquer ce qui a motivé leur décision et ce qu'ils attendent de ce projet. Dans l'édition n ${ }^{\circ} 10$ du Bulletin des médecins suisses parue le 7 mars dernier, vous avez pu prendre connaissance des expériences des représentants de la psychiatrie et de la médecine de famille, qui soutiennent le projet depuis ses débuts en 2010.

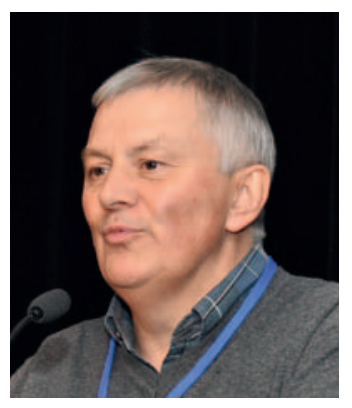

Jacques Seydoux

Président de la Société Suisse de Gynécologie et d'Ostétrique SSGO.

«A l'instar des autres disciplines, la gynécologie et l'obstétrique portent un grand intérêt au développement de la qualité. Grâce au projet Monitorage de la qualité, le comité de la SSGO peut désormais contribuer activement à ce développement en prenant connaissance des activités qualité menées aujourd'hui déjà par ses confrères et de leur fréquence. Ces informations nous permettront non seulement de consolider notre stratégie interne en matière de qualité mais également de disposer de données solides pour étayer les arguments du corps médical dans le débat sur la qualité mené avec nos partenaires du secteur de la santé et le monde politique. C'est pourquoi j'invite tous les gynécologues $\mathrm{du}$ domaine ambulatoire et tous nos collègues du domaine ambulatoire des autres disciplines impliquées dans ce projet à participer à l'enquête!»

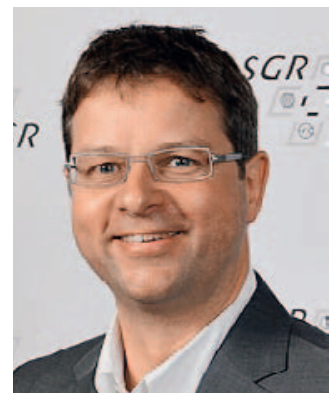

Thomas Böhm

Chef du Domaine Qualité de la Société suisse de radiologie SSR

«La Société suisse de radiologie a décidé de rejoindre le projet Monitorage de la qualité, qui représente selon elle un pas dans la bonne direction en offrant davantage de visibilité à l'engagement des médecins du domaine ambulatoire pour la qualité. Avec les neuf autres sociétés de discipline impliquées dans le projet, nous espérons récolter des données représentatives et solides - ce qui est essentiel pour faire entendre notre voix! Grâce au Monitorage de la qualité, nous avons également l'occasion de rencontrer des collègues d'autres disciplines et d'unir nos forces afin d'améliorer ensemble la qualité des soins. Comme l'a montré la collaboration au sein de l'équipe de projet, les activités qualité ne sont souvent pas mises en œuvre dans une seule et unique discipline, mais dans les disciplines les plus diverses.» 


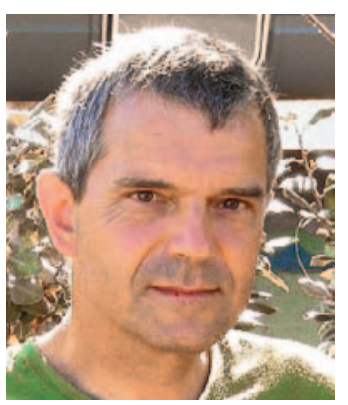

\section{Peter Wiederkeh}

Responsable du domaine Qualité de la Société suisse d'anesthésiologie et de réanimation SSAR.

«Pour une discipline comme l'anesthésiologie et la réanimation, dans laquelle seul un tiers des spécialistes travaille dans le domaine ambulatoire, la participation au projet Monitorage de la qualité permet de compléter idéalement les projets du domaine hospitalier. Désormais, les anesthésistes du domaine ambulatoire ont eux aussi la possibilité de rendre visible leur engagement quotidien en faveur de la qualité. Par la même occasion, le comité de la SSAR va pouvoir développer sa stratégie en matière de qualité tout en tenant compte des particularités du domaine ambulatoire. Pour toutes ces raisons, vous êtes tous cordialement invités à participer à l'enquête!»

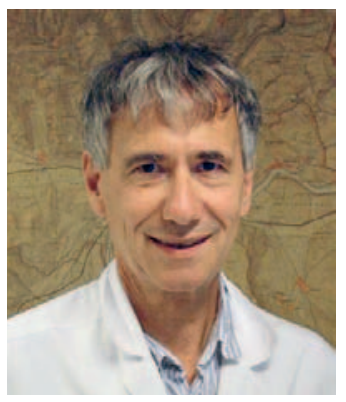

\section{Jürg Häggi}

Responsable du domaine Qualité de la Société suisse de pneumologie SSP.

«Le Monitorage de la qualité ne profite pas seulement à la Société suisse de pneumologie, mais à tous nos collègues du domaine ambulatoire. L'analyse des données permettra à chaque participant d'obtenir un précieux retour sur ses activités qualité et d'identifier les domaines dans lesquels il est déjà très actif et ceux dans lesquels il doit intensifier ses efforts. Grâce à ce projet, participants et sociétés de discipline auront toutes les clés en main pour poursuivre le développement de l'assurance-qualité dans la pratique.»

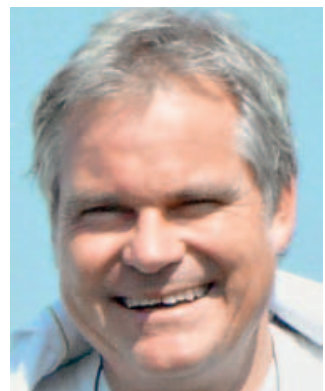

\section{Florian Lang}

Responsable du domaine Qualité de la Société suisse d'oto-rhino-laryngologie et de chirurgie cervico-faciale SSORL.
«Le projet Monitorage de la qualité n'est pas un outil caché devant permettre à la FMH de contrôler, de juger ou de condamner l'activité médicale du praticien installé. Il ne sert pas non plus à mesurer la qualité de nos traitements ambulatoires! Ce projet doit nous permettre d'obtenir une vue d'ensemble des nombreuses activités qualité déjà effectuées par les praticiens, de manière à mieux les mettre en évidence. Il doit également démontrer quels types d'initiatives qualité pourraient encore être utilement implémentés. C'est seulement à l'aide d'une argumentation chiffrée que la Société suisse d'ORL sera en mesure d'effectuer à l'interne une analyse pertinente de la situation. Le but est de permettre à ses membres de se déterminer démocratiquement quant à l'utilité d'introduire d'éventuelles nouvelles activités qualité. Il faut également des chiffres pour pouvoir se comparer à d'autres sociétés médicales et pour mener des discussions de politique professionnelle.»

\section{Participer à l'enquête}

Vous êtes spécialiste dans I'une des disciplines suivantes:

- Médecine interne générale*

Anesthésiologie*

- Gynécologie et obstétrique*

- Pédiatrie*

- Psychiatrie et psychothérapie de l'enfant et de l'adolescent ${ }^{* *}$

- Oto-rhino-laryngologie*

- Pneumologie**

- Psychiatrie et psychothérapie**

- Radiologie*

* L'enquête se poursuit jusqu'au 4 avril 2012

** L'enquête se poursuit jusqu'au 2 mai 2012
Nous invitons tous les médecins qui le souhaitent à déclarer leurs activités en faveur de la qualité dès aujourd'hui, de manière strictement confidentielle. En participant à I'enquête, vous aiderez votre société de discipline à élaborer et à développer sa propre stratégie en matière de qualité. Vous aurez également la possibilité de comparer vos activités avec celles de l'ensemble de vos confrères et contribuerez ainsi à renforcer la crédibilité du corps médical dans ce domaine en améliorant durablement la transparence de ses activités!

Vous trouverez les questionnaires à l'adresse www.myfmh.ch. Nous sommes à votre entière disposition pour toute question sur le projet ou le questionnaire, par courriel à ddq[at]fmh.ch ou par téléphone au 031 35911 11. Si vous avez des questions sur la plateforme myFMH, et en particulier sur l'enregistrement, notre service d'administration des membres y répondra volontiers, par téléphone au 0313591259 ou par courriel à myfmh[at]fmh.ch. Vous trouverez de plus amples informations sur le projet Monitorage de la qualité en médecine ambulatoire dans l'ensemble de la Suisse sur www.fmh.ch $\rightarrow$ Autres thèmes $\rightarrow$ Qualité à Monitorage.

Les sociétés de discipline impliquées et la $\mathrm{FMH}$ vous remercient d'ores et déjà de votre précieuse collaboration! 